\title{
High spatial resolution free-breathing 3D late gadolinium enhancement cardiac magnetic resonance imaging in ischaemic and non-ischaemic cardiomyopathy: quantitative assessment of scar mass and image quality
}

\author{
Maurice B. Bizino ${ }^{1} \cdot$ Qian Tao $^{1} \cdot$ Jacob Amersfoort ${ }^{1} \cdot$ Hans-Marc J. Siebelink $^{2} \cdot$ Pieter J. van den Bogaard $^{1} \cdot$ Rob J. van \\ der Geest ${ }^{1} \cdot$ Hildo J. Lamb ${ }^{1}$
}

Received: 25 August 2017 / Revised: 13 January 2018 / Accepted: 30 January 2018 / Published online: 6 April 2018

(C) The Author(s) 2018

\begin{abstract}
Purpose To compare breath-hold (BH) with navigated free-breathing (FB) 3D late gadolinium enhancement cardiac MRI (LGECMR)

Materials and methods Fifty-one patients were retrospectively included (34 ischaemic cardiomyopathy, 14 non-ischaemic cardiomyopathy, three discarded). BH and FB 3D phase sensitive inversion recovery sequences were performed at 3T. FB datasets were reformatted into normal resolution (FB-NR, 1.46x1.46x10mm) and high resolution (FB-HR, isotropic 0.91-mm voxels). Scar mass, scar edge sharpness (SES), SNR and CNR were compared using paired-samples $t$-test, Pearson correlation and Bland-Altman analysis.

Results Scar mass was similar in BH and FB-NR (mean \pm SD: $15.5 \pm 18.0 \mathrm{~g}$ vs. $15.5 \pm 16.9 \mathrm{~g}, p=0.997$ ), with good correlation $(\mathrm{r}=0.953)$, and no bias (mean difference $\pm \mathrm{SD}: 0.00 \pm 5.47 \mathrm{~g})$. FB-NR significantly overestimated scar mass compared with FBHR (15.5 $\pm 16.9 \mathrm{~g}$ vs $14.4 \pm 15.6 \mathrm{~g} ; p=0.007)$. FB-NR and FB-HR correlated well ( $\mathrm{r}=0.988)$, but Bland-Altman demonstrated systematic bias $(1.15 \pm 2.84 \mathrm{~g})$. SES was similar in BH and FB-NR $(p=0.947)$, but significantly higher in FB-HR than FB-NR $(p<0.01)$. SNR and CNR were lower in BH than FB-NR $(p<0.01)$, and lower in FB-HR than FB-NR $(p<0.01)$.

Conclusion Navigated free-breathing 3D LGE-CMR allows reliable scar mass quantification comparable to breath-hold. During free-breathing, spatial resolution can be increased resulting in improved sharpness and reduced scar mass.

Key Points

- Navigated free-breathing 3D late gadolinium enhancement is reliable for myocardial scar quantification.

- High-resolution $3 D$ late gadolinium enhancement increases scar sharpness

- Ischaemic and non-ischaemic cardiomyopathy patients can be imaged using free-breathing LGE CMR.
\end{abstract}

Keywords Late gadolinium enhancement, MRI · High spatial resolution · Myocardial infarction · Non-ischaemic cardiomyopathy $\cdot$ Free-breathing

Electronic supplementary material The online version of this article (https://doi.org/10.1007/s00330-018-5361-y) contains supplementary material, which is available to authorized users.

Maurice B. Bizino

m.b.bizino@lumc.nl

1 Department of Radiology, Leiden University Medical Center, Albinusdreef 2, 2333 ZA Leiden, The Netherlands

2 Department of Cardiology, Leiden University Medical Center, Albinusdreef 2, 2333 ZA Leiden, The Netherlands

$\begin{array}{ll}\text { Abbreviations } \\ \text { BH } & \text { Breath-hold } \\ \text { BMI } & \text { Body mass index } \\ \text { CNR } & \text { Contrast-to-noise ratio } \\ \text { FA } & \text { Flip angle } \\ \text { FB-HR } & \text { High spatial resolution free-breathing } \\ \text { FB-NR } & \text { Normal spatial resolution free-breathing } \\ \text { FB } & \text { Free-breathing } \\ \text { FOV } & \text { Field of view } \\ \text { FWHM } & \text { Full width half maximum } \\ \text { ICM } & \text { Ischaemic cardiomyopathy }\end{array}$




$\begin{array}{ll}\text { IR } & \text { Inversion recovery } \\ \text { L G E - } & \text { Late gadolinium enhancement cardiac magnetic } \\ \text { CMR } & \text { resonance imaging } \\ \text { MRI } & \text { Magnetic resonance imaging } \\ \text { NICM } & \text { Non-ischaemic cardiomyopathy } \\ \text { PSIR } & \text { Phase-sensitive inversion recovery } \\ \text { SD } & \text { Standard deviation } \\ \text { SENSE } & \text { Sensitivity encoding } \\ \text { SES } & \text { Scar edge sharpness } \\ \text { SI } & \text { Signal intensity } \\ \text { SNR } & \text { Signal-to-noise ratio } \\ \text { TE } & \text { Echo time } \\ \text { TI } & \text { Inversion time } \\ \text { TR } & \text { Repetition time }\end{array}$

\section{Introduction}

Late gadolinium enhancement (LGE) imaging is an established cardiovascular magnetic resonance (CMR) technique to assess myocardial viability [1]. LGE-CMR is commonly used in the diagnosis of ischaemic cardiomyopathy (ICM) and to guide revascularisation therapy. In addition, LGE-CMR has the ability to detect partially infarcted myocardial tissue, known as grey zone, which is believed to be a substrate for arrhythmia [2]. Grey zone mass quantification using LGE-CMR has been shown to predict post-myocardial infarction mortality [3]. In non-ischaemic cardiomyopathy (NICM), a specific LGE pattern allows for a differential diagnosis [1]. More recently, scar mass quantification has been shown to predict adverse cardiovascular outcome in various non-ischaemic cardiomyopathies [4-6]. The increasing range of clinical applications warrants optimisation of current LGECMR protocols.

Standard LGE-CMR is performed during multiple breathholds in several pre-defined two-dimensional (2D) orientations with an inversion recovery (IR) gradient echo sequence with the inversion time manually chosen to null the signal of healthy myocardium [1]. Three-dimensional (3D) LGE-CMR is desirable because it allows (1) imaging of the whole heart without slice gaps, (2) imaging with high SNR and CNR, and (3) performing post-acquisition reformatting in any desired plane $[7,8]$. However, 3D LGE-CMR is hampered by long breath-hold duration ( $>20 \mathrm{~s}$ ) [9], thereby compromising its robustness in a clinical setting that includes vulnerable patients [10]. To overcome these limitations, 3D LGE-CMR can be performed during free-breathing, for example with respiratory gating based on diaphragmatic pencil-beam navigation [7]. The free-breathing approach allows to apply a phase sensitive inversion recovery sequence (PSIR) in combination with high spatial resolution (submillimeter voxel size). The PSIR sequence triggers inversions every other heartbeat with an additional proton density-weighted reference image acquired on alternate heartbeats. As a result, PSIR reconstructed images are less sensitive to wrong inversion time and heart rate variability as compared with standard inversion recovery [7]. In theory, high spatial resolution LGE-CMR should result in better defined scar borders (specified as scar edge sharpness, SES), which could diminish partial volume averaging that contributes to scar and grey zone mass overestimation $[11,12]$. To date, breath-hold 3D LGE-CMR has not been compared with free-breathing 3D LGE-CMR with matched spatial resolution. Furthermore, high spatial resolution freebreathing 3D LGE-CMR has not been compared with normal spatial resolution free-breathing 3D LGE-CMR in a clinical setting.

Therefore, the purpose of this study was to compare scar mass, grey zone mass, SES, and image quality of late gadolinium enhancement cardiac MRI using multiple breath-hold 3D phase sensitive inversion recovery imaging with matched normal spatial resolution navigated free-breathing 3D LGECMR (FB-NR), and to compare FB-NR with high spatial resolution navigated free-breathing 3D LGE-CMR (FB-HR) at 3.0 Tesla in ischaemic and non-ischaemic cardiomyopathy patients.

\section{Methods}

\section{Ethics}

Navigated free-breathing LGE-CMR validation against standard breath-hold LGE-CMR was performed within the frame of clinical CMR protocol development. This retrospective study was considered a chart review. Therefore, written informed consent was waived by the hospital institutional review board.

\section{Patient population}

From May 2012 until November 2013, breath-hold and freebreathing sequences were acquired in patients that were referred for LGE-CMR, if there was enough time in the clinical scanning slot. Of the 54 patients in which both $\mathrm{BH}$ and $\mathrm{FB}$ were performed, there were two incomplete free-breathing datasets (one acquisition was stopped due to allergic contrast reaction; one was stopped because of very low navigator efficiency). In one patient there was no LGE detectable. The remaining 51 patients were included (38 male; 13 female; mean age \pm standard deviation: 59.9 years \pm 11.4 years).

\section{MRI}

All CMR examinations were performed in our institution on a 3T unit (Ingenia, Philips Healthcare, Best, The Netherlands) with the patient in supine position. The body coil was used for 
transmission, and a 16-element anterior and 12-element posterior phased-array coil were used for signal reception. Following a standard clinical CMR protocol including 2D cine imaging in all cardiac axis views, the gadolinium-based contrast agent gadoterate meglumine (Dotarem, Guerbet, Villepinte, France) was administered intravenously at a dose of $0.15 \mathrm{mmol} / \mathrm{kg}$. Ten minutes after contrast administration, a 2D gradient-echo T1 weighted sequence was used (i.e. LookLocker) to visually determine the optimal inversion time (TI) of healthy myocardium. Approximately $10-15 \mathrm{~min}$ postcontrast, a whole heart high spatial resolution 3D gradient echo (T1 fast field echo) PSIR sequence was acquired during free breathing with diaphragmatic pencil-beam navigation. Image parameters were as follows: repetition time (TR) $4.15 \mathrm{~ms}$; echo time (TE) $2.02 \mathrm{~ms}$; TI was set at the null point plus $50 \mathrm{~ms}$ and ranged from 250 to $400 \mathrm{~ms}$; flip angle (FA) $10^{\circ}$; field of view (FOV) $350 \times 350 \mathrm{~mm}$; matrix size $208 \times 208$; acquired pixel size $1.68 \times 1.68$ $\mathrm{mm}$; reconstructed pixel size $0.91 \times 0.91 \mathrm{~mm} ; 71$ transverse slices with $3.4 \mathrm{~mm}$ thickness and slice gap $-1.7 \mathrm{~mm}$; sensitivity encoding (SENSE) factor 3. The 2D pencil beam respiratory navigator was planned on the right hemidiaphragm. The acceptance window was set at $5 \mathrm{~mm}$ with a constant correction of 0.6 . Saturation bands were planned on the navigator, vertebrae and thoracic subcutaneous adipose tissue to suppress residual motion artefacts. Acquisition time was 3 min $4 \mathrm{~s}$ assuming $100 \%$ navigator efficiency and heart rate of 60 beats per minute (bpm). Directly after free-breathing LGE-CMR, BH was acquired using a 3D gradient echo PSIR sequence in short-axis slice orientation in two equal stacks during two breath-holds of 10-20 s duration. Subsequently, left two-chamber and four-chamber cardiac views were acquired using the same sequence. Typical scan parameters were: TR $4.31 \mathrm{~ms}$; TE $2.09 \mathrm{~ms}$; TI 250-400 ms (same as free-breathing); flip angle $10^{\circ}$; FOV $350 \times 350 \mathrm{~mm}$; matrix size $188 \times 125 \mathrm{~mm}$; acquired pixel size $1.86 \times 2.8$ $\mathrm{mm}$; reconstructed pixel size $1.46 \times 1.46 \mathrm{~mm}$; 24 slices with $10 \mathrm{~mm}$ thickness and $-5 \mathrm{~mm}$ slice gap; SENSE factor 3 .

\section{Image analysis}

MASS research software (Department of Radiology, Leiden University Medical Centre, Leiden, The Netherlands) was used for multiplanar reformatting and image analysis. $\mathrm{BH}$ data were analysed after offline fusion of data of the two breath-hold 3D stacks. Free-breathing 3D datasets were reformatted into two multiplanar reconstructions with identical geometry as $\mathrm{BH}$ : one with normal spatial resolution (FB-NR: $1.46 \times 1.46 \times 10 \mathrm{~mm}$ ), matching BH, and one with high spatial resolution isotropic voxel reconstruction (FBHR: $0.91 \times 0.91 \times 0.91 \mathrm{~mm}$ ). (See Online Supplementary
Material for a description of reconstruction method.) Images were analysed in random order. First, endocardial and epicardial contours were drawn manually. Second, regions with maximally hyperenhanced and hypoenhanced healthy myocardium were identified visually and a small region of interest was drawn. Third, myocardial scar was identified automatically using the validated full-width half maximum (FWHM) algorithm [2, 13, 14]. For this algorithm, maximal signal intensity $\left(\mathrm{SI}_{\max }\right)$ was defined as the mean signal intensity of the manually annotated hyperenhanced region, and minimal signal intensity $\left(\mathrm{SI}_{\min }\right)$ was defined as the mean signal intensity of the manually annotated hypoenhanced region. Myocardial scar was defined as voxels with signal intensity higher than $50 \%$ $\left(\mathrm{SI}_{\min }+0.5 \times\left(\mathrm{SI}_{\max }-\mathrm{SI}_{\min }\right)\right.$. Grey zone (only reported for ICM patients) was defined as voxels with signal intensity between $35 \%\left(\mathrm{SI}_{\min }+0.35 \times\left(\mathrm{SI}_{\max }-\mathrm{SI}_{\min }\right)\right.$ and $50 \%$ $\left(\mathrm{SI}_{\min }+0.5 \times\left(\mathrm{SI}_{\max }-\mathrm{SI}_{\min }\right)\right.$. Fourth, manual adjustments to the automatically determined areas of scar were made only in cases of evident misinterpretation by the algorithm, for example in case of microvascular obstruction. Finally, scar and grey zone masses were calculated (in grams: specific weight of scar was set at $1.05 \mathrm{~g} / \mathrm{ml}$ ) using an in-house developed MATLAB script (Version R2012A, Natick, MA, USA).

SNR was calculated by dividing $\left(\mathrm{SI}_{\max }-\mathrm{SI}_{\text {reference }}\right)$ by the noise (standard deviation of signal outside the body). $\mathrm{SI}_{\text {max }}$ was subtracted by $\mathrm{SI}_{\text {reference }}$ to compensate for high signal intensity of the nulled healthy myocardium in PSIR (which is 0 in magnitude inversion recovery images) [15]. CNR was computed as the difference between $\mathrm{SI}_{\max }$ and $\mathrm{SI}_{\text {min }}$, divided by the noise (standard deviation of signal outside the body). Figure 1 is a $2 \mathrm{D}$ representation of $3 \mathrm{D}$ computation of SES, which was computed with an algorithm in the Matlab environment (v. R2012a, Mathworks, Natick, MA, USA). To account for differences in signal intensity between breath-hold and free-breathing datasets, the signal intensity of all voxels containing myocardium were normalised on a scale of $0 \%\left(\mathrm{SI}_{\min }\right)$ to $100 \%$ $\left(\mathrm{SI}_{\max }\right): \mathrm{SI}_{\text {normalised }}=\left(\mathrm{SI}-\mathrm{SI}_{\min }\right) /\left(\mathrm{SI}_{\max }-\mathrm{SI}_{\min }\right)$. Then, at the edge of the scar, the steepness of the slope (expressed in $\triangle \mathrm{SI}_{\text {normalised }} / \mathrm{mm}$ ) reflects SES. A SES of 1.0 reflects a full transition of scar to healthy myocardium over a $1.0-\mathrm{mm}$ distance.

\section{Statistical analysis}

Data are presented as mean \pm standard deviation. $\mathrm{BH}$ sequence was compared to FB-NR for validation purposes. Subsequently, FB-NR was compared with FB-HR. Outcome measures were compared using paired-samples $t$-test. Agreement of scar mass was compared using Pearson's correlation coefficient and Bland Altman 


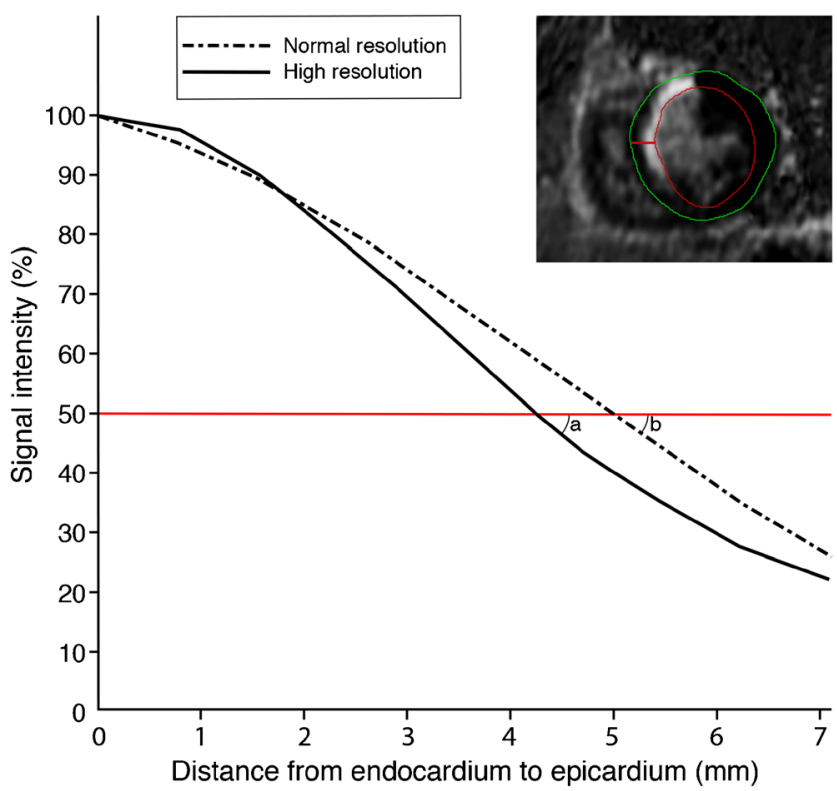

Fig. 1 Computation of scar edge sharpness. On the upper right side of this figure a short-axis slice of a patient with anteroseptal myocardial infarction is displayed. In this slice, the red line perpendicular to the endocardial border represents the transition from dense subendocardial scar to healthy myocardium subepicardially. The graph illustrates the signal intensity alongside the red line (dotted line represents normal resolution freebreathing LGE-CMR; continuous line represents high resolution freebreathing LGE-CMR). The border of the scar is defined by the points where the red horizontal line crosses the signal intensity curves. The steepness of the slope at the scar border gives rise to the scar edge sharpness (SES), expressed in $\Delta \mathrm{SI} / \mathrm{mm}$. In this figure, note that the steepness is higher in high resolution (angle $a$ ) as compared with normal resolution (angle $b$ ). It is important to appreciate that the gradient was calculated for the $3 \mathrm{D}$ volumes instead of the $2 \mathrm{D}$ representation of this figure

analysis. A $p$ value of less than 0.05 was considered to indicate statistical significance. Statistical analyses were performed using SPSS software (version 20, SPSS, Chicago, IL, USA).

\section{Results}

Of the 51 patients included, three had free-breathing images of insufficient quality due to poor ECG-triggering, breathing artefact and absence of clear myocardial scar, respectively. The remaining 48 patients were analysed ( 36 male; 12 female; age 60.8 years \pm 10.9 years; BMI $27.6 \mathrm{~kg} / \mathrm{m}^{2} \pm 5.1 \mathrm{~kg} / \mathrm{m}^{2}$ ), of which 34 had ICM (seven acute, four subacute, 23 chronic; 27 male; seven female; age 61.1 years \pm 8.4 years; BMI 28.3 $\mathrm{kg} / \mathrm{m}^{2} \pm 5.1 \mathrm{~kg} / \mathrm{m}^{2}$ ) and 14 had NICM (dilated cardiomyopathy $\mathrm{n}=5$; hypertrophic cardiomyopathy $\mathrm{n}=4$; acute myocarditis $\mathrm{n}=3$; other $\mathrm{n}=2 ; 9$ male; 5 female; age 59.5 years \pm 15.6 years; BMI $24.8 \mathrm{~kg} / \mathrm{m}^{2} \pm 4.3 \mathrm{~kg} / \mathrm{m}^{2}$ ). Mean acquisition time ( \pm $\mathrm{SD}$ ) was $4 \min 22 \mathrm{~s} \pm 1 \min 25 \mathrm{~s}$ for BH LGE-CMR (all three cardiac views) and $9 \min 34 \mathrm{~s} \pm 3 \min 4 \mathrm{~s}$ for free-breathing
LGE-CMR. Figures 2, 3 and 4 show examples of BH, FB-NR and FB-HR images in patients with ICM and NICM. Figure 5 shows a normal versus high spatial resolution 3D reconstruction of the free-breathing dataset.

\section{Scar mass, all patients $(n=48)$}

Scar mass was the same in BH versus FB-NR (mean \pm SD: $15.5 \mathrm{~g} \pm 18.0 \mathrm{~g}$ vs. $15.5 \mathrm{~g} \pm 16.9 \mathrm{~g}, p=0.997)$. Moreover, scar mass showed good correlation $(\mathrm{r}=0.953)$ without systematic bias in Bland Altman analysis (mean difference \pm SD: $0.00 \mathrm{~g} \pm$ $5.47 \mathrm{~g}$ ), as shown in the Online Supplementary Material. Limits of agreement were between $-10.94 \mathrm{~g}$ and $+10.93 \mathrm{~g}$.

FB-NR yielded higher scar mass as compared with FB-HR (15.5 $\mathrm{g} \pm 16.9 \mathrm{~g}$ vs. $14.4 \mathrm{~g} \pm 15.6 \mathrm{~g} ; p=0.007)$. Although the data correlated well $(r=0.988)$, Bland Altman analysis demonstrated a systematic positive bias of $+1.15 \mathrm{~g}$ for FB-NR versus FB-HR (mean difference \pm SD: $+1.15 \mathrm{~g} \pm 2.84 \mathrm{~g}$ ), as shown in the Online Supplementary Material. Limits of agreement were between $-4.42 \mathrm{~g}$ and $+6.72 \mathrm{~g}$.

\section{Scar and grey zone mass ICM patients $(n=34)$}

In the ICM subgroup, scar mass was $18.9 \mathrm{~g} \pm 20.2 \mathrm{~g}$ in BH versus $19.3 \mathrm{~g} \pm 18.7 \mathrm{~g}$ in FB-NR, which was not significantly different $(p=0.670)$. In parallel with scar mass in all patients, FB-NR overestimated scar mass as compared with FB-HR in the ICM subgroup $(19.3 \mathrm{~g} \pm 18.7 \mathrm{~g}$ vs. $17.9 \mathrm{~g} \pm 17.2 \mathrm{~g}, p=$ 0.01 ).

Grey zone was not significantly different between $\mathrm{BH}$ versus FB-NR (6.5 $\mathrm{g} \pm 5.4 \mathrm{~g}$ vs. $6.0 \mathrm{~g} \pm 6.0 \mathrm{~g}, p=0.128)$, and between FB-NR vs. FB-HR $(6.0 \mathrm{~g} \pm 6.0 \mathrm{~g}$ vs. $6.2 \mathrm{~g} \pm 6.0 \mathrm{~g}$, $p=0.329$ ).

\section{Scar mass NICM patients $(n=14)$}

In NICM patients scar mass was similar in BH vs. FB-NR (7.3 $\pm 6.1 \mathrm{~g}$ vs. $6.3 \mathrm{~g} \pm 4.3 \mathrm{~g}, p=0.312$ ). The difference in scar mass between FB-NR and FB-HR was not significant in this subgroup $(6.3 \mathrm{~g} \pm 4.3 \mathrm{~g}$ vs. $5.9 \mathrm{~g} \pm 4.8 \mathrm{~g}, p=0.457)$.

\section{Image quality}

As shown in the Online Supplementary Material, SNR and CNR were significantly lower in $\mathrm{BH}$ as compared with FBNR $(207.6 \pm 85.8$ vs. $1052.2 \pm 877.2, p<0.01$ and $221.9 \pm$ 70.9 vs. $864.9 \pm 692.5$, respectively). As a result of higher spatial resolution, SNR and CNR decreased significantly in FB-HR as compared with FB-NR (258.3 \pm 94.9 vs. $1052.2 \pm$ $877.2, p<0.01$ and $232.4 \pm 91.5$ vs. $864.9 \pm 692.5, p<0.01$, respectively). In the ICM subgroup, SNR and CNR were lower in BH as compared with FB-NR (224.7 \pm 87.3 vs. $1125.0 \pm$ $1010.9, p<0.01$ and $240.4 \pm 66.8$ vs. $935.1 \pm 789.1, p<0.01$, 
Fig. 2 Example of patient with ischaemic cardiomyopathy. A 58year-old man with acute myocardial infarction of the anterior wall due to obstruction in the left anterior descending artery for which primary percutaneous revascularisation was performed. Two weeks later LGE-CMR was performed. Transmural LGE of the anteroseptal wall with areas without LGE centrally in the scar can be depicted (no reflow zones). The horizontally aligned images have the exact same orientation and position in the heart and show close agreement with respect to scar visualisation. Note the sharpness of the high spatial resolution images (right panel) characterised by detailed borders of scar and areas of microvascular obstruction, as compared with normal spatial resolution (middle panel) and breath-hold (left panel) images
Fig. 3 Example of a patient with perimyocarditis. A 49-year-old man who presented with thoracic pain related to breathing and posture. The electrocardiogram showed PR depression and ST elevation. CMR was performed for diagnostic purposes. LGECMR shows two focal subepicardial areas of enhancement (apical - mid inferior, and mid - basal anterior), as well as diffuse pericardial enhancement. It can be appreciated that the borders of scar are less blurry in the highresolution images. Also note that the right ventricular myocardium, and surrounding pericardial enhancement can be identified best in the high-resolution images
Breath-hold
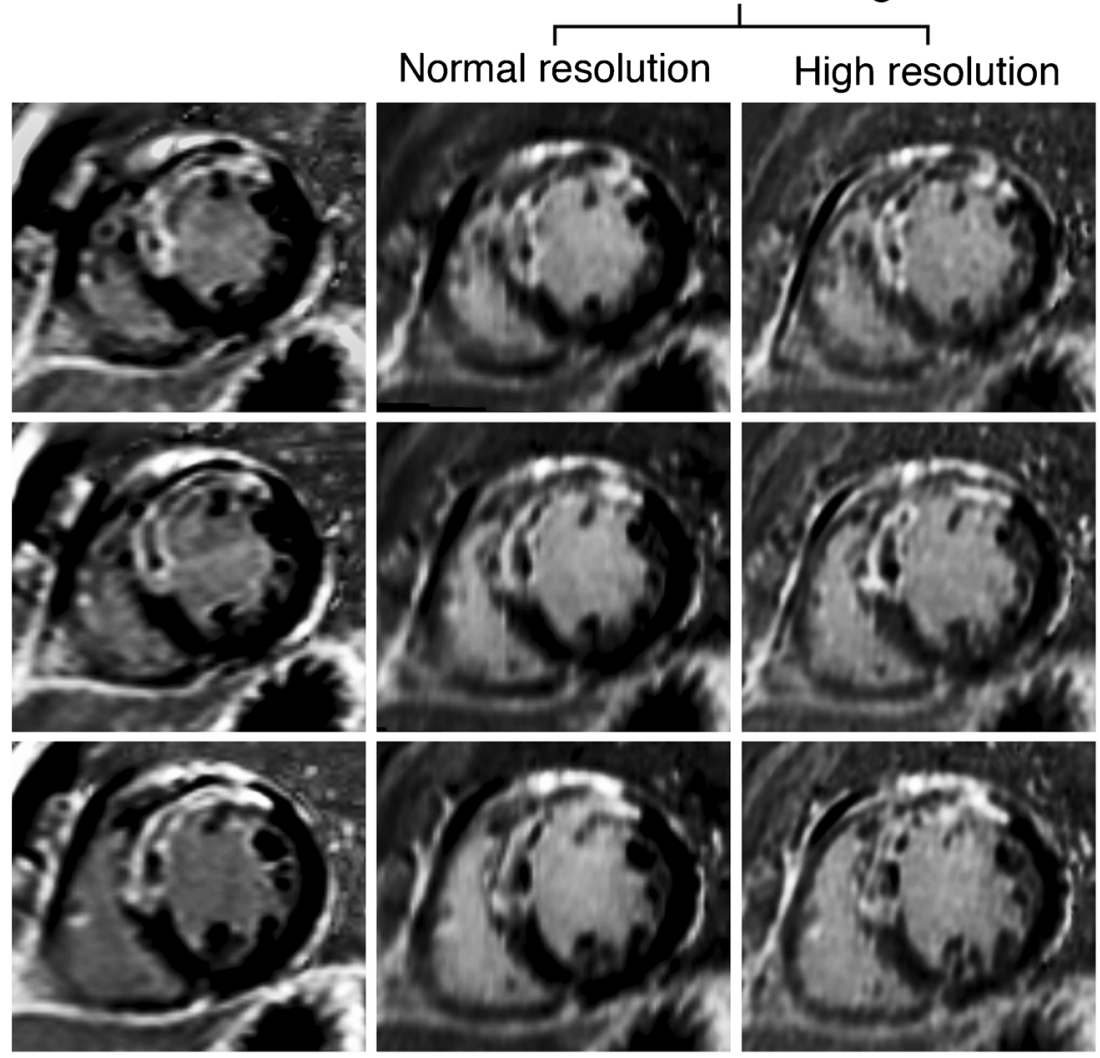
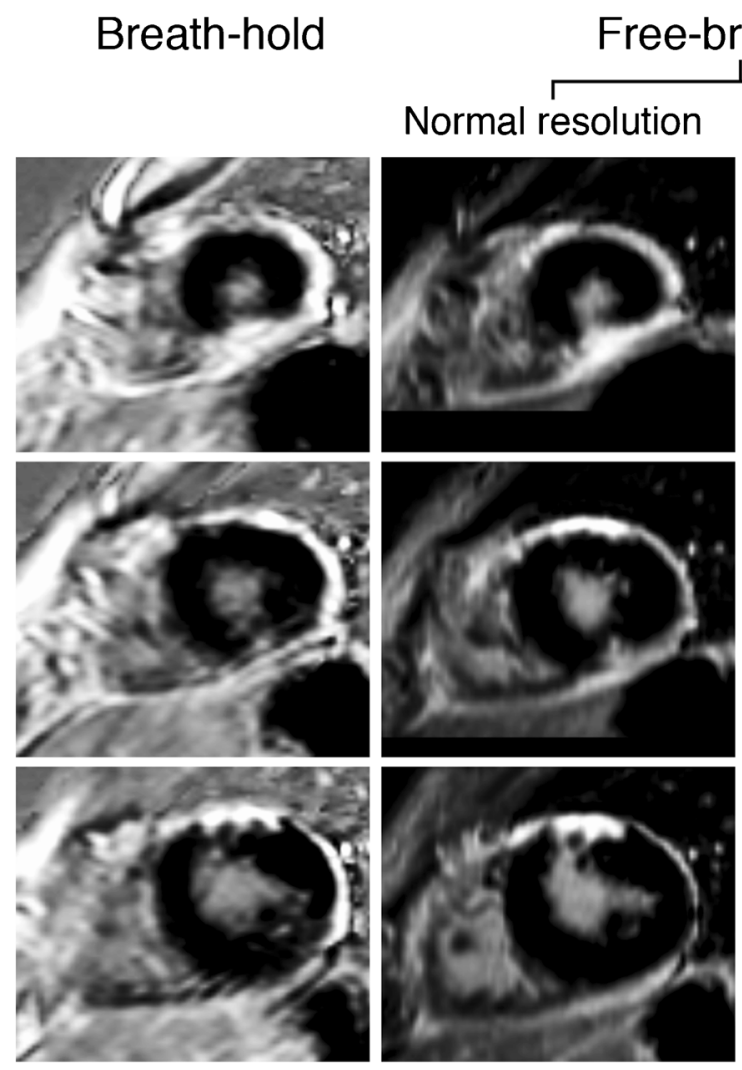
Fig. 4 Example of a patient with hypertrophic cardiomyopathy. A 48-year-old woman with hypertrophic cardiomyopathy and non-sustained ventricular tachycardias originated from the interventricular septum. LGECMR images display asymmetrical septal hypertrophy and focal lesions of contrast enhancement at the right ventricular insertion point. Note close agreement of LGE area and volume in the images aligned horizontally. Furthermore, the sharpness of high spatial resolution images (right panel) is very high as reflected by the less blurry transition from scar to healthy myocardium as compared with the left and middle panel. Also note that there is less partial volume erroring of the right ventricular wall in high resolution images as compared with breathhold and free-breathing normal resolution

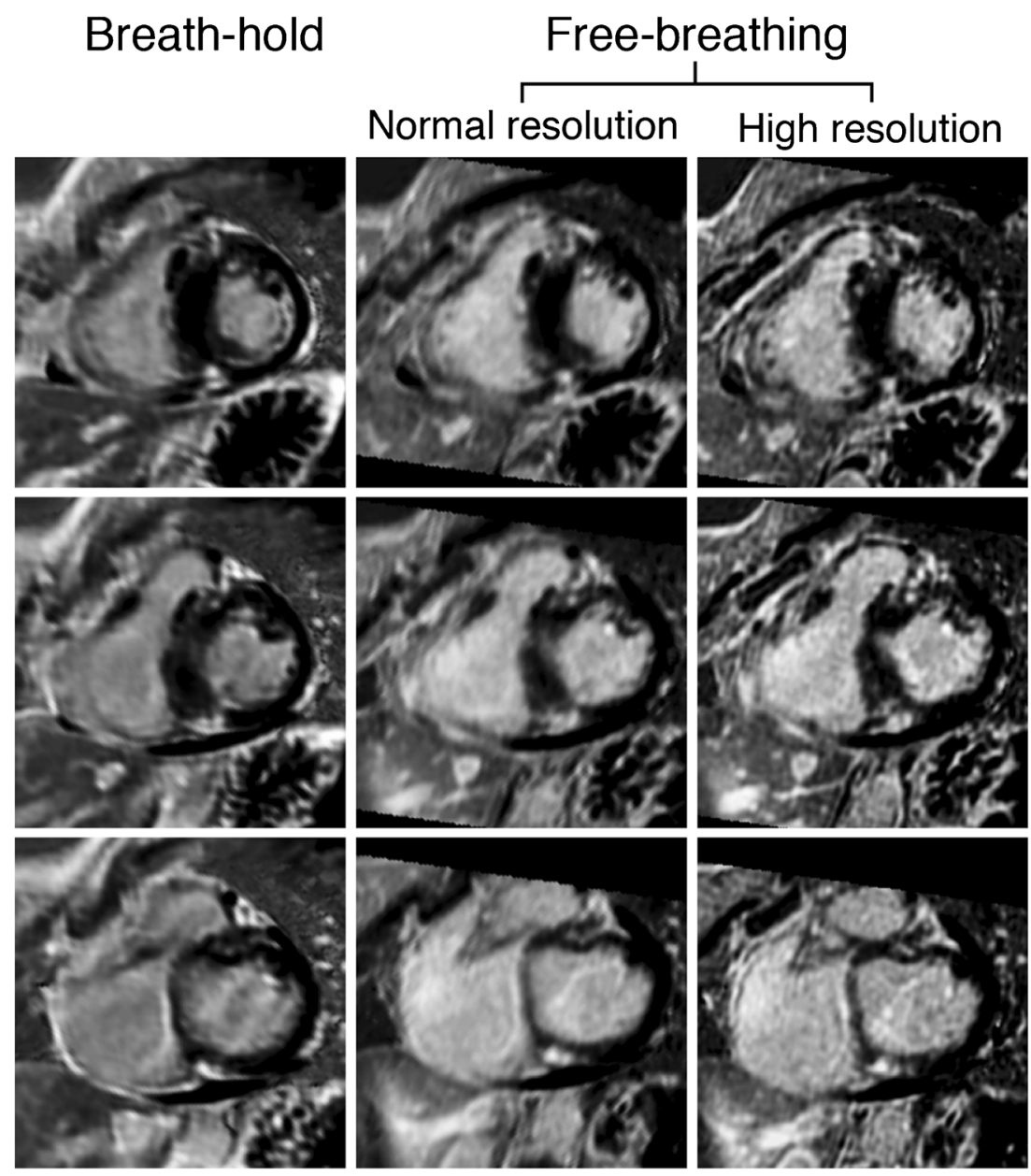

respectively). In FB-HR, SNR and CNR were lower as compared with FB-NR $(272.9 \pm 93.4$ vs. $1125.0 \pm 1010.9, p<0.01$ and $250.6 \pm 89.1$ vs. $935.1 \pm 789.1, p<0.01$, respectively). Also in the NICM subgroup, SNR and CNR were lower in $\mathrm{BH}$ as compared with FB-NR (165.9 \pm 67.9 vs. $875.5 \pm 374.4, p<$ 0.01 and $177.1 \pm 61.4$ vs. $694.3 \pm 330.3, p<0.01$, respectively), and lower in FB-HR as compared to FB-NR $(222.8 \pm 92.1$ vs. $875.5 \pm 374.4, p<0.01$ and $188.2 \pm 84.5$ vs. $694.3 \pm 330.3$, $p<0.01$, respectively).

SES was similar in $\mathrm{BH}$ versus FB-NR $(0.176 \pm 0.039$ vs. $0.176 \pm 0.036, p=0.947)$, see Online Supplementary Material. FB-HR images had significantly higher SES as compared to FB-NR $(0.216 \pm 0.042$ vs. $0.176 \pm 0.036, p<0.01)$. The absolute increase in sharpness was 0.040 with $95 \%$
Fig. 5 Normal versus high spatial resolution $3 \mathrm{D}$ reconstruction of left ventricle. Both a normal spatial resolution (left panel, 1.68 $\times 1.68 \times 10 \mathrm{~mm})$ and high spatial resolution (right panel, $0.91 \times$ $0.91 \times 0.91 \mathrm{~mm}) 3 \mathrm{D}$ reconstruction were generated from the free-breathing dataset of the patient represented in Fig. 3 . This reconstruction shows the sharper delineated scar border in the high spatial resolution image

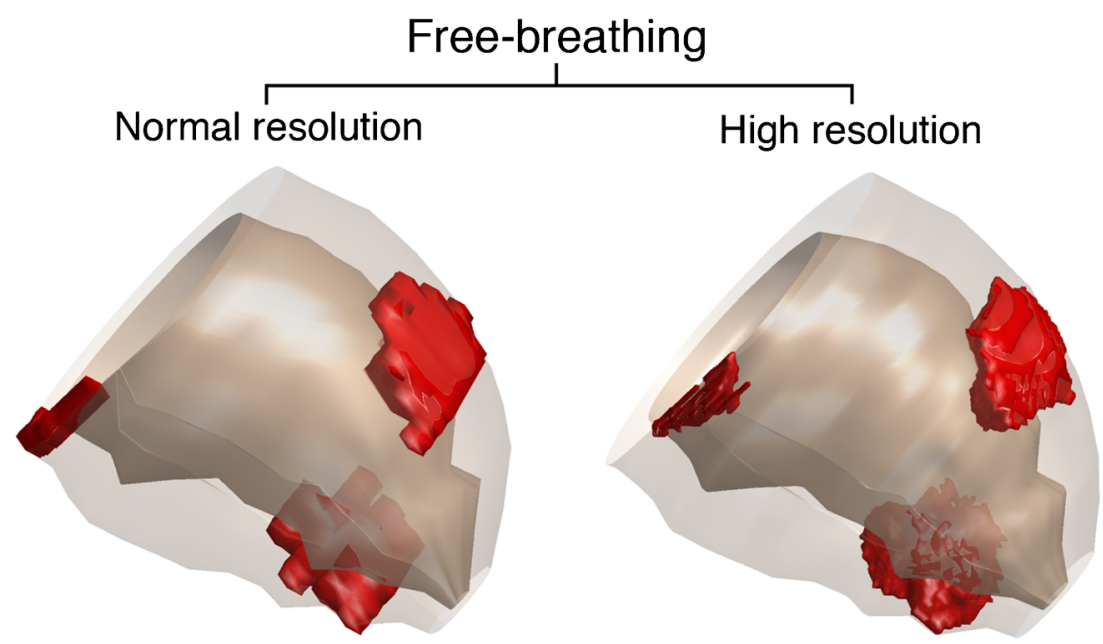


confidence interval between 0.032 and 0.048 , corresponding to a relative increase in SES of $22.7 \%$ with $95 \%$ confidence interval between 18.7 and 27.2. An example of the different signal intensity profiles across the edge of the scar of FB-NR vs. FB-HR is shown in Fig. 6. In the ICM subgroup, SES was the same in $\mathrm{BH}$ and FB-NR $(0.176 \pm 0.033$ vs. $0.173 \pm 0.036$, $p=0.639)$, and statistically significantly higher in FB-HR as compared with FB-NR $(0.212 \pm 0.038$ vs. $0.173 \pm 0.036, p<$ 0.01). Also in the NICM subgroup, SES was the same in $\mathrm{BH}$ and FB-NR $(0.176 \pm 0.053$ vs. $0.182 \pm 0.039, p=0.460)$, and statistically significantly higher in FB-HR as compared with FB-NR $(0.227 \pm 0.050$ vs. $0.182 \pm 0.039, p<0.01)$.

\section{Discussion}

This study demonstrates that, as compared with multiple breath-hold 3D LGE-CMR, navigated free-breathing 3D LGE-CMR with matched spatial resolution allows reliable scar mass quantification with good image quality. Additionally, the free-breathing technique allows to image with high spatial resolution, thereby improving scar edge sharpness and reducing scar mass. This study involves ischaemic and non-ischaemic cardiomyopathy patients in a

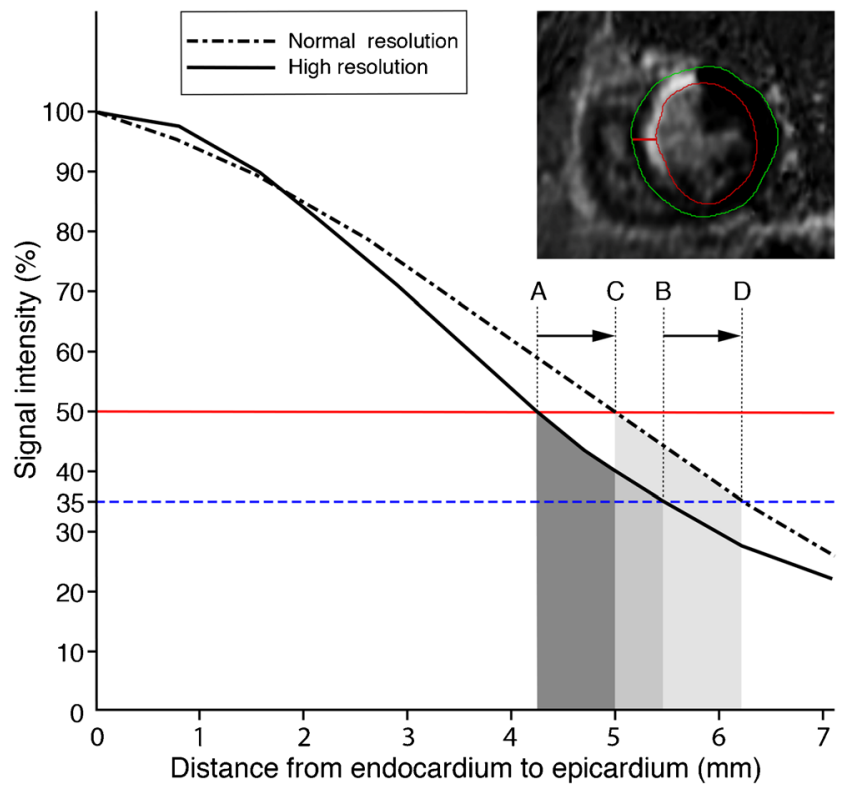

Fig. 6 Scar and grey zone mass in relation to spatial resolution. This figure represents data of a patient with anteroseptal myocardial infarction (same patient as in Fig. 1). The signal intensity profile alongside the red line depicted in the short axis image is plotted in the graph. In the normal resolution image (dotted black line), the transition of scar to healthy myocardium is less steep as compared with high resolution (continuous line), resulting in a larger scar mass (shift from A to C). The outer grey zone border at $35 \%$ signal intensity (horizontal blue dotted line) also shifts $(\mathrm{A} \rightarrow \mathrm{C}=\mathrm{B} \rightarrow \mathrm{D})$. Therefore, grey zone mass (illustrated by the grey areas between $35 \%$ and $50 \%$ signal intensity) is not larger in normal resolution (light grey area) as compared with high spatial resolution (dark grey area) LGE-CMR clinical setting which emphasises that the proposed sequence could be considered an alternative for breath-hold LGE-CMR at $3 \mathrm{~T}$ systems.

Traditionally, LGE-CMR has been performed during multiple breath-holds. Breath-holds frequently cause slice misregistration, and repeated breath-holding can be challenging for vulnerable cardiomyopathy patients [10]. This study shows that free-breathing 3D LGE-CMR yields higher SNR and CNR, as compared with breath-hold LGE-CMR with matched spatial resolution. Importantly, FB-NR allows reliable scar and grey zone mass quantification for ICM and NICM patients in a clinical setting on a 3 Tesla MR scanner. The reallife clinical setting also included vulnerable patients: population comprised 10 patients over 70 years of age, and several patients with heart failure and/or large transmural myocardial scar. Subgroup analysis of patients under and over 60 years of age revealed that there was no difference regarding image quality parameters between young and old patients (data not shown). Piehler et al. have found comparable results of motion-corrected free-breathing 2D PSIR sequence compared with a breath-hold 2D PSIR sequence with matched spatial resolution at 1.5 Tesla [10]. This study extends their findings to the 3D-PSIR acquisition technique at 3 Tesla. Schultz et al. have compared breath-hold 3D-PSIR with 3D-IR LGE-CMR at $3 \mathrm{~T}$ showing superior image quality of the former sequence [16]. To our knowledge, this study is the first to compare a breath-hold 3D-PSIR sequence with a free-breathing 3DPSIR sequence, with matched spatial resolution.

Because breath-holds can be challenging for patients, spatial resolution in breath-hold LGE-CMR is generally in the range of 5-10 $\mathrm{mm}$ in the through-plane direction. By relieving patients from repeated breath-holding, navigated freebreathing 3D LGE-CMR allows to image the whole heart without slice gaps with higher spatial resolution (especially in the through-plane direction). Our data show that, in FBHR images, the scar border is better delineated (i.e. less blurry), as compared with FB-NR, supported by a $22.7 \%$ relative increase in SES. Despite the increase in spatial resolution, SNR and CNR remained within the range of $\mathrm{BH}$ images. While others have shown subjective improvement of image sharpness of a high spatial resolution free-breathing 3D sequence against a normal spatial resolution breath-hold 2D sequence at 1.5 Tesla [17], our study is the first to objectively quantify SES in LGE-CMR. As shown in Fig. 6, we propose that increased SES in FB-HR results in smaller scar mass by reducing partial volume effects. Jablonowski et al. have shown that normal spatial resolution LGE-CMR overestimates scar mass, as compared with a high spatial resolution ex vivo sequence [11]. As such, it may be assumed that FBHR reduces scar mass overestimation. Aforementioned study by Peters et al. found no difference in scar mass between a high spatial resolution $(0.62 \times 0.62 \times 2.5 \mathrm{~mm})$ free-breathing $3 \mathrm{D}$ inversion recovery sequence and a normal spatial 
resolution $(1.2 \times 1.2 \times 8 \mathrm{~mm})$ breath-hold $2 \mathrm{D}$ inversion recovery sequence in 14 ICM patients [17]. The discrepancy between our results and theirs might be explained by our larger difference in spatial resolution and different cohort size. Furthermore, we used two reconstructions from the same 3D dataset instead of two separately acquired sequences. By doing so, other variables than voxel size in itself are eliminated. Scar mass was not significantly smaller in FB-HR than FBNR in the NICM subgroup analysis. This lack of difference is possibly related to the small sample size, and the fact that the FWHM method has lower reproducibility in NICM as compared with ICM [5].

Contrary to scar mass, grey zone mass did not differ between FB-NR and FB-HR. As illustrated in Fig. 6, the difference between the signal intensity decay of FB-NR and FB-HR diminishes at the transition area of scar to grey zone. As a result, the difference in grey zone mass is reduced to a non-significant level. It is important to note that the region characterised as grey zone probably shifts towards the endocardium in FB-HR. This suggest that FB-HR might localize grey zone more precisely. Schelbert et al. have performed ex vivo LGE-CMR with spatial resolutions ranging from the cellular level to approximately the level of our FBHR reconstruction [12]. They found a positive linear correlation between grey zone mass and voxel size, explained by a reduction in partial volume averaging at the border of definite scar and healthy myocardium. However, whether their results in the ex vivo rat heart can be extrapolated to in vivo human clinical resolution images remains uncertain. Another discrepancy with our study is the method used for grey zone quantification. The FWHM method used in our study has been shown to have higher reproducibility as compared with the standard deviation threshold method used by Schelbert et al. [2, 13].

From a clinical perspective, navigated free-breathing 3D LGE-CMR has the advantage to relieve patients from repeated breath-holding and generate images with superior image quality. In theory, high spatial resolution LGE-CMR could enable more accurate visualisation of specific patterns of (very) small scar lesions, especially critical in the case of NICM. In ICM, sharper delineation of scar might better define degree of transmurality, or define the location of grey zone area, for example to improve pre-procedural guidance of ablation therapy in arrhythmia patients. Furthermore, in a research setting, scar mass quantification is often applied in (non-) ICM to correlate with adverse cardiovascular outcome. This study shows that results from LGE-CMR protocols with varying spatial resolutions should be interpreted with caution. Also, application of a high spatial resolution free-breathing 3D LGE-CMR might increase power of clinical studies that include LGE-CMR. Finally, the longer scan duration of high spatial resolution free-breathing 3D LGE-CMR must be taken into account. The scan duration of $9-10 \mathrm{~min}$ might be challenging for the most vulnerable or critically ill patients. In such settings, voxel size might be reduced. Alternatively, the number of slices could be reduced to include only the left ventricle instead of the whole heart. It needs to be stressed that a single free-breathing 3D LGE dataset allows reformatting in any desired plane and scan duration should be compared with acquisition of multiple $2 \mathrm{D}$ views.

\section{Limitations}

Our study has several limitations. First, the reference standard of scar mass quantification is histopathological staining which was not possible to perform in this study. Second, the nonrandom order of sequences could have compromised SNR and CNR in breath-hold LGE-CMR (which was always performed after free-breathing LGE-CMR) due to contrast washout. However, both sequences were performed with PSIR compensating for suboptimal TI selection due to wash out $[18,19]$. In our view, the four- to fivefold increase of SNR and CNR could not be explained by the scan order. Another limitation is the retrospective character of the study which did not allow evaluating the robustness of the proposed sequence.

\section{Conclusion}

In a clinical setting including ischaemic and non-ischaemic cardiomyopathy patients, normal spatial resolution navigated free-breathing 3D LGE-CMR allows reliable scar mass quantification with higher SNR and CNR as compared with multiple breath-hold 3D LGE-CMR. High spatial resolution freebreathing 3D LGE-CMR has improved scar edge sharpness and reduced scar mass, as compared with normal spatial resolution free-breathing 3D LGE-CMR. High spatial resolution free-breathing 3D LGE-CMR might improve scar mass quantification and diagnostic performance, whilst relieving the patient from repeated breath-holding. In addition, free-breathing 3D LGE-CMR allows post-acquisition reformatting in any desired imaging plane.

Acknowledgements We would to thank Gerrit Kracht for editing the figures.

Funding The authors state that this work has not received any funding.

\section{Compliance with ethical standards}

Guarantor The scientific guarantor of this publication is Prof H.J. Lamb.

Conflict of interest The authors of this manuscript declare no relationships with any companies whose products or services may be related to the subject matter of the article. 
Statistics and biometry No complex statistical methods were necessary for this paper.

Informed consent Written informed consent was waived by the Institutional Review Board.

Ethical approval Institutional Review Board approval was not required because the institution has stated that informed consent was waived because all scans were performed within clinical work-up.

\section{Methodology}

- retrospective

- observational

- performed at one institution

Open Access This article is distributed under the terms of the Creative Commons Attribution 4.0 International License (http:// creativecommons.org/licenses/by/4.0/), which permits unrestricted use, distribution, and reproduction in any medium, provided you give appropriate credit to the original author(s) and the source, provide a link to the Creative Commons license, and indicate if changes were made.

\section{References}

1. Ordovas KG, Higgins CB (2011) Delayed contrast enhancement on MR images of myocardium: past, present, future. Radiology 261(2):358-374

2. Roes SD, Borleffs CJ, van der Geest RJ et al (2009) Infarct tissue heterogeneity assessed with contrast-enhanced MRI predicts spontaneous ventricular arrhythmia in patients with ischemic cardiomyopathy and implantable cardioverter-defibrillator. Circ Cardiovasc Imaging 2(3):183-190

3. Yan AT, Shayne AJ, Brown KA et al (2006) Characterization of the peri-infarct zone by contrast-enhanced cardiac magnetic resonance imaging is a powerful predictor of post-myocardial infarction mortality. Circulation 114(1):32-39

4. Chan RH, Maron BJ, Olivotto I et al (2014) Prognostic value of quantitative contrast-enhanced cardiovascular magnetic resonance for the evaluation of sudden death risk in patients with hypertrophic cardiomyopathy. Circulation 130(6):484-495

5. Gao P, Yee R, Gula L et al (2012) Prediction of arrhythmic events in ischemic and dilated cardiomyopathy patients referred for implantable cardiac defibrillator: evaluation of multiple scar quantification measures for late gadolinium enhancement magnetic resonance imaging. Circ Cardiovasc Imaging 5(4):448-456

6. Kuruvilla S, Adenaw N, Katwal AB et al (2014) Late gadolinium enhancement on cardiac magnetic resonance predicts adverse cardiovascular outcomes in nonischemic cardiomyopathy: a systematic review and meta-analysis. Circ Cardiovasc Imaging 7(2):250-258
7. Kellman P, Arai AE (2012) Cardiac imaging techniques for physicians: late enhancement. J Magn Reson Imaging 36(3):529-542

8. Kido T, Kido T, Nakamura M et al (2014) Three-dimensional phase-sensitive inversion recovery sequencing in the evaluation of left ventricular myocardial scars in ischemic and non-ischemic cardiomyopathy: comparison to three-dimensional inversion recovery sequencing. Eur J Radiol 83(12):2159-2166

9. Goetti R, Kozerke S, Donati OF et al (2011) Acute, subacute, and chronic myocardial infarction: quantitative comparison of $2 \mathrm{D}$ and 3D late gadolinium enhancement MR imaging. Radiology 259(3): 704-711

10. Piehler KM, Wong TC, Puntil KS et al (2013) Free-breathing, motion-corrected late gadolinium enhancement is robust and extends risk stratification to vulnerable patients. Circ Cardiovasc Imaging 6(3):423-432

11. Jablonowski R, Nordlund D, Kanski M et al (2013) Infarct quantification using $3 \mathrm{D}$ inversion recovery and $2 \mathrm{D}$ phase sensitive inversion recovery; validation in patients and ex vivo. BMC Cardiovasc Disord 13:110

12. Schelbert EB, Hsu LY, Anderson SA et al (2010) Late gadoliniumenhancement cardiac magnetic resonance identifies postinfarction myocardial fibrosis and the border zone at the near cellular level in ex vivo rat heart. Circ Cardiovasc Imaging 3(6):743-752

13. Amado LC, Gerber BL, Gupta SN et al (2004) Accurate and objective infarct sizing by contrast-enhanced magnetic resonance imaging in a canine myocardial infarction model. J Am Coll Cardiol 44(12):2383-2389

14. Rajchl M, Stirrat J, Goubran M et al (2015) Comparison of semiautomated scar quantification techniques using high-resolution, 3dimensional late-gadolinium-enhancement magnetic resonance imaging. Int J Cardiovasc Imaging 31(2):349-357

15. Stirrat J, Joncas SX, Salerno M et al (2015) Influence of phase correction of late gadolinium enhancement images on scar signal quantification in patients with ischemic and non-ischemic cardiomyopathy. J Cardiovasc Magn Reson 17(1):66

16. Schultz A, Caspar T, Schaeffer M et al (2016) Late gadolinium enhancement cardiac imaging on a $3 \mathrm{~T}$ scanner with parallel RF transmission technique: prospective comparison of 3D-PSIR and 3D-IR. Eur Radiol 26:1547-1555

17. Peters DC, Appelbaum EA, Nezafat R et al (2009) Left ventricular infarct size, peri-infarct zone, and papillary scar measurements: A comparison of high-resolution $3 \mathrm{D}$ and conventional 2D late gadolinium enhancement cardiac MR. J Magn Reson Imaging 30(4): 794-800

18. Wagner A, Mahrholdt $\mathrm{H}$, Thomson $\mathrm{L}$ et al (2006) Effects of time, dose, and inversion time for acute myocardial infarct size measurements based on magnetic resonance imaging-delayed contrast enhancement. J Am Coll Cardiol 47(10):2027-2033

19. Wildgruber M, Settles M, Kosanke K et al (2012) Evaluation of phase-sensitive versus magnitude reconstructed inversion recovery imaging for the assessment of myocardial infarction in mice with a clinical magnetic resonance scanner. J Magn Reson Imaging 36(6): $1372-1382$ 